\title{
c-Jun, at the crossroad of the signaling network
}

\author{
Qinghang Meng, Ying Xia \\ Department of Environmental Health, University of Cincinnati, College of Medicine, Cincinnati, OH 45267, USA \\ \ Correspondence: ying.xia@uc.edu \\ Received October 6, 2011 Accepted October 11, 2011
}

\begin{abstract}
c-Jun, the most extensively studied protein of the activator protein-1 (AP-1) complex, is involved in numerous cell activities, such as proliferation, apoptosis, survival, tumorigenesis and tissue morphogenesis. Earlier studies focused on the structure and function have led to the identification of c-Jun as a basic leucine zipper (bZIP) transcription factor that acts as homo- or heterodimer, binding to DNA and regulating gene transcription. Later on, it was shown that extracellular signals can induce post-translational modifications of c-Jun, resulting in altered transcriptional activity and target gene expression. More recent work has uncovered multiple layers of a complex regulatory scheme in which c-Jun is able to crosstalk, amplify and integrate different signals for tissue development and disease. One example of such scheme is the autocrine amplification loop, in which signal-induced AP-1 activates the c-Jun gene promoter, while increased c-Jun expression feedbacks to potentiate AP-1 activity. Another example of such scheme, based on recent characterization of gene knockout mice, is that c-Jun integrates signals of several developmental pathways, including EGFR-ERK, EGFR-RhoA-ROCK, and activin B-MAP3K1-JNK for embryonic eyelid closure. After more than two decades of extensive research, c-Jun remains at the center stage of a molecular network with mysterious functional properties, some of which are yet to be discovered. In this article, we will provide a brief historical overview of studies on c-Jun regulation and function, and use eyelid development as an example to illustrate the complexity of c-Jun crosstalking with signaling pathways.
\end{abstract}

KEYWORDS mitogen-activated protein kinase kinase kinase 1 (MAP3K1), c-Jun amino-terminal kinases (JNKs), activator protein-1 (AP-1), gene transcription, phosphorylation

\section{c-Jun, A MEMBER OF THE AP-1 COMPLEX}

The Jun protein was originally identified in transformed cells carrying the genome of a replication defective avian sarcoma virus 17 (ASV 17) that directs the expression of a $65-\mathrm{kDa}$ gag-jun fusion product, designated as v-Jun (Maki et al., 1987; Bos et al., 1988). Soon after, its homolog, the proto-oncogene c-Jun, was isolated from human and murine tissues (Bohmann et al., 1987; Ryder et al., 1988). cJun shares sequence similarity not only with $v$-jun, but also with the yeast transcription regulatory protein GCN4. GCN4 and the mammalian AP-1, known at that time only as a transcription factor interacting with a specific enhancer sequence, share the same DNA binding specificity (Short, 1987; Haluska et al., 1988). Using this specific piece of DNA for affinity chromatography, the mammalian c-Jun was co-purified with c-Fos, marking the first identification of AP-1 as a heterodimer of C-Jun and C-Fos (Angel et al., 1988a; Harshman et al., 1988; Rauscher et al., 1988a, 1988b).

The AP-1 family has been greatly expanded in the past two decades. The current view of AP-1 is a collective term for dimers formed by proteins of the Jun (C-Jun, JunB and JunD), Fos (c-Fos, FosB, Fra1 and Fra2), activating transcription factor (ATF) (ATF2, ATF3/LRF1, B-ATF, JDP1 and JDP2) and musculoaponeurotic fibrosarcoma (MAF) (c-Maf, MafB, MafA, MafG/F/K and Nrl) families (Hai et al., 1988; Angel and Karin, 1991). These are structurally similar and functionally related basic leucine zipper (bZIP) proteins, which form homo- and/or hetero-dimers through the bZIP domain. Dimerization brings together the basic regions, producing a 
contiguous DNA-contact interface that interacts with specific sequences of DNA (Ellenberger et al., 1992; Schumacher et al., 2000). Once bound to DNA, the AP-1 complexes function as transcription regulators to either activate or repress target gene transcription.

The seemingly simple regulatory scheme, however, is entangled due to the complexity of the intrinsic properties and regulation of the AP-1 proteins (Karin et al., 1997). First, each AP-1 member has unique dimerization capacity. While the Jun proteins form homo- or hetero-dimers with members of Fos and ATF families, ATF, but not Fos, also form stable homodimers. On the other hand, the c-Maf and $\mathrm{Nrl}$ can heterodimerize with both c-Jun and c-Fos, but other Mafrelated proteins, including MafB, MafF, MafG and MafK, form dimers with only Fos, but not Jun (Landschulz et al., 1988). Second, albeit all dimers bind to DNA with the consensus 5'TGA(C/G)TCA-3' sequence, each dimer has different binding affinities and sequence specificities. For example, Jun/ATF preferentially binds to the CAMP-responsive element (CRE, $5^{\prime}$-TGACGTCA-3'), while Jun/Fos has high affinity for the phorbol 12 O-tetradecanoate-13-acetate (TPA)-responsive element (TRE, 5'-TGAG/CTCA-3'). Some dimers also exhibit weak affinity for DNA sequences that deviate from the consensus. Third, the AP-1 proteins can interact with proteins outside the family, resulting in complexes with unique DNA binding and transcription activity. Examples for this type of interaction include c-Jun binding to the Ets domain of PU.1, directing the complex to the PU.1 binding site in the $\mathrm{M}-\mathrm{CSF}$ receptor promoter for transcriptional activation (Behre et al., 1999), whereas, the c-Jun/MyoD complex is recruited to the MyoD promoter to inhibit myogenesis (Bengal et al., 1992).

Another layer of complexity comes from considerable variations in AP-1 expression and activity. Each AP-1 component exhibits unique cell type- and tissue-specific distribution (Wagner et al., 2010), but this can be modified by the cellular environment. A vast number of growth factors, cytokines, chemokines, hormones and environmental stresses have been shown to regulate AP-1 at the level of gene expression, mRNA turnover and protein stability, thereby affecting the relative abundance of AP-1 within the cells. In addition, many of these signals can lead to modifications of AP-1 proteins, changing their abilities of forming dimers, binding to DNA and activating gene transcription.

Collectively, AP-1 exists as a complex mixture of dimers with different DNA binding specificities and transcriptional activity, and is able to regulate expression of a large number of genes. As a result, AP-1 has been implicated in a wide range of cellular processes, including proliferation, death, survival and differentiation, and participates in diverse biological and pathological processes, such as epidermal and neuronal development, immune and inflammatory responses, and tumorigenesis (Shaulian and Karin, 2002).

\section{c-Jun EXPRESSION AND ACTIVITY}

C-Jun is the founding member and the most potent transcriptional activator of the AP-1 family (Angel and Karin, 1991). It has been shown that tumor promoter phorbol-ester (TPA), growth factors or stress stimuli can significantly enhance the DNA binding and transcription activity of c-Jun (Lee et al., 1987; Quantin and Breathnach, 1988; Ryder and Nathans, 1988; Pertovaara et al., 1989; Sherman et al., 1990; Devary et al., 1991). Initiation of this process begins with stimulus activated signal transduction cascades that lead to a rapid change in the phosphorylation state of c-Jun. This event takes place on the pre-existing c-Jun, within minutes of stimulus treatment, and is independent of de novo protein synthesis (Lamph et al., 1988; Bohmann, 1990; Papavassiliou et al., 1992).

Phosphorylation can occur on at least five serine and threonine residues of C-Jun (Boyle et al., 1991; Baker et al., 1992). Three phosphorylation sites are located just upstream of the basic region in the DNA-binding domain (residues 227-252); stimuli, such as TPA, can decrease the C-terminal domain phosphorylation thereby increasing the DNA binding affinities of c-Jun. The other two phosphorylation sites are located within the $\mathrm{N}$-terminal transcription activation domain at serines 63 and 73. TPA significantly increases the $\mathrm{N}$ terminal phosphorylation, resulting in a robust induction of cJun transactivation activity (Smeal et al., 1991; Lin et al., 1992; Smeal et al., 1992; Nikolakaki et al., 1993). In contrast, Jun B, lacking serine-63 and-73, is irresponsive to TPA induced transcriptional activation (Franklin et al., 1992).

While phosphorylation of c-Jun may provide a kick start for AP-1 activation, the phospho-C-Jun themselves are relatively unstable and exist only transiently in the cells (Black et al., 1991; Franklin et al., 1995). This feature makes it unlikely for $\mathrm{p}$-c-Jun to maintain the sustained AP-1 activation required for many of the biological activities (Behrens et al., 1999; Behrens et al., 2000). On the other hand, AP-1 activity is closely associated with a steady elevation of c-Jun expression (Devary et al., 1991). Detailed analyses of the C-Jun promoter identify binding sites for NF-KB, sp1, CCAATbinding transcription factors, but more interestingly, the promoter contains a high-affinity AP-1 binding site. Mutation of this site abolishes the induction of c-Jun mRNA (Angel et al., 1988b). These observations suggest the existence of a regulatory circuit, in which AP-1 can activate the c-Jun promoter and gene expression, whereas, expression of c-Jun in turn further enhances AP-1 thereby potentiating its own gene promoter activation (Fig. 1) (Angel et al., 1988b; Lamph et al., 1988; Nakamura et al., 1991). Such an autocrine and feed-forward mechanism allows c-Jun to not only prolong AP1 activity, but also amplify it. As a result, c-Jun can efficiently convert transient biochemical signals to sustained AP-1 activity and act as a potent transcription factor to regulate long-lasting biological outcomes (Angel et al., 1988b). 
A

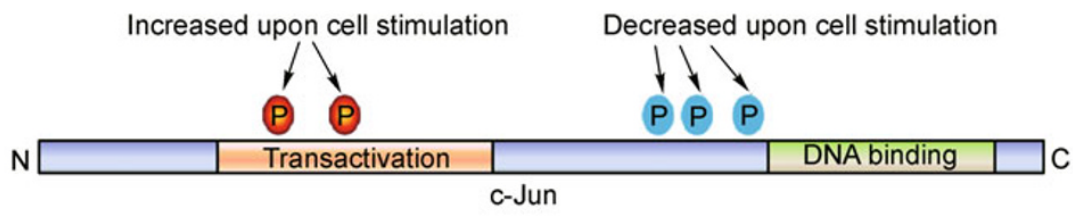

B

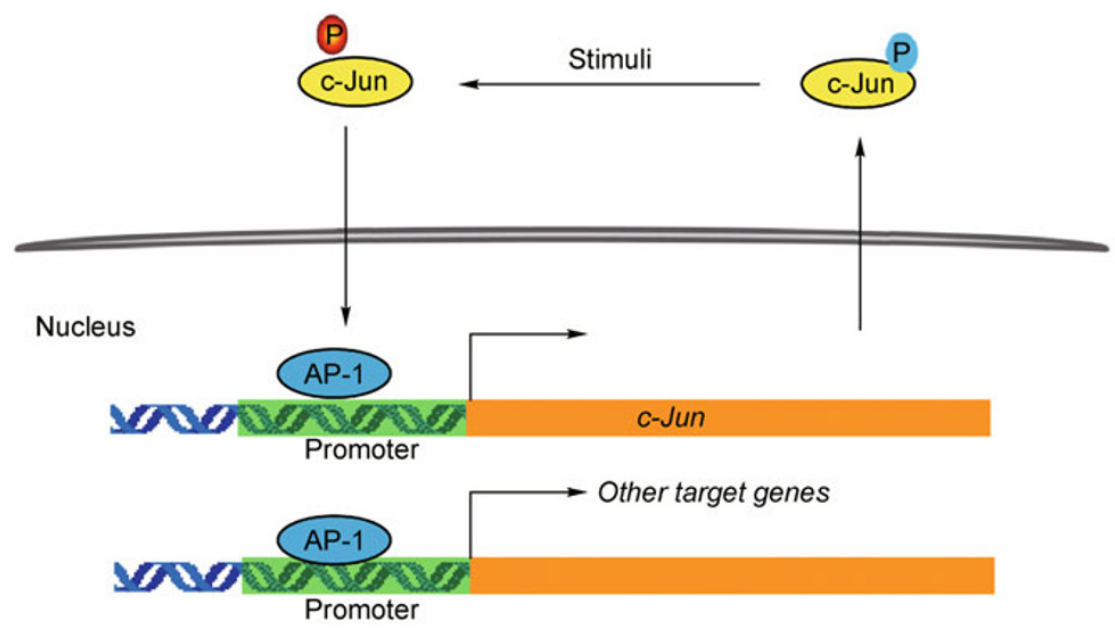

Figure 1. Regulation of c-Jun expression and activity. A diagram illustrating (A) the phosphorylation sites on c-Jun and (B) the autocrine amplification loop between c-Jun expression and AP-1 activity.

\section{SIGNALING MECHANISMS OF c-Jun PHOSPHORYLATION}

Transmission of the extracellular signals through the cytoplasm is mediated by cascades of protein kinases that ultimately lead to phosphorylation of transcription factors (Bohmann, 1990; Karin, 1991). Glycogen synthase kinase 3 (GSK-3) and CKII can phosphorylate c-Jun at its C-termini, keeping c-Jun in a non DNA-binding state (Boyle et al., 1991), while extracellular signal regulated kinase (ERK) can activate p70 S6 kinase, which in turn phosphorylates GSK-3 at serine21, resulting in its inactivation (Sutherland et al., 1994). Hence, ERK, by acting through the p70 S6 kinase-GSK-3 cascades, causes c-Jun C-terminal dephosphorylation and increases its DNA binding activity. A functional connection between ERK and c-Jun has been established by mutation studies in fission yeast, in which homologs of mammalian ERK and Jun act in concert to control yeast cell elongation immediately after division (Toda et al., 1991).

The c-Jun amino-terminal kinases (JNKs) have been identified based on their activation by UV and oncoproteins (Hibi et al., 1993; Dérijard et al., 1994). Like the ERKs, the JNKs are proline-directed kinases, preferentially phosphorylate serine and threonine located within the Pro-Xaa-Ser/ Thr-Pro sequences. However, the JNKs have distinct substrate specificities, as they do not phosphorylate p70 S6 kinase, but phosphorylate efficiently c-Jun at the $\mathrm{N}$-terminal domain. JNK phosphorylates c-Jun at serines 63 and 73 and phosphorylation requires binding of JNK to a specific region within the c-Jun transactivation domain (Hibi et al., 1993; Dérijard et al., 1994; Kyriakis et al., 1994). This characteristic explains why v-Jun, having completely conserved Ser-63 to Ser-73, but lacking the JNK binding domain (amino acids 34-60), is resistant to TPA-induced N-terminal phosphorylation (Adler et al., 1992).

The JNKs and the ERKs, together with the later discovered p38s, constitute three separate groups of mitogen-activated protein kinases (MAPKs). The MAPKs themselves are activated through concomitant phosphorylation on tyrosine and threonine residues in the Thr-Xxx-Tyr motif. For each MAPK group, the phosphorylation is catalyzed by the designated MAP kinase kinases (MAPKKs), a novel class of dual specific protein kinases (Mordret, 1993). Activation of each MAPK depends on specific upstream MAPKKs. The MAPKKs MEK4 and MEK7 activate the JNKs, the MEK1 and MEK2 activate the ERK, and the MEK3 and MEK6 activate the p38 (Johnson and Lapadat, 2002). Activation of the MAPKKs is in turn catalyzed by their immediate upstream kinases, the MAPKK kinases (MAPKKKs), which phosphorylate the MAPKKs at serine/threonine residues (Davis, 1994).

In less than 20 years since the discovery of its first member, the MAPKKK superfamily has expanded to more than 20 protein kinases, including Rafs, MEK kinase 1-4 (MAP3K 1-4), germinal center kinase (GCK), mixed lineage kinases 
(MLK), apoptosis-stimulated kinase 1(ASK1), tumor progression locus 2 (TPL2), and TGF-beta-activated kinase (TAK). While all MAPKKKs have distinct regulatory domains, which are used to connect to the specific upstream regulators, they have relatively similar kinase domains, which are used to activate the MAPKK-MAPK cascades. Thus, each MAPKKK is believed responsible for mediating specific upstream cues and the family collectively is able to connect diverse signals to the MAPKK-MAPK cascades (Fig. 2) (Schlesinger et al., 1998).

The most convincing evidence supporting this view comes from genetic ablation of individual MAPKKK in mice, which display distinct phenotypes. Some of the knockout mice, including Raf-1(-l-), Map3k3(-l-) and Map3k4(-l-), die in embryogenesis but of different causes (Johnson et al., 1993; Kuan et al., 1999). The Raf-1(-/-) fetuses show vascular defects in the yolk sac and placenta as well as increased apoptosis of embryonic tissues (Hüser et al., 2001), the Map3k3(-l-) embryos die due to impaired blood vessels development (Yang et al., 2000), while the Map3k4(-l-) mice die from neural tube defects associated with massively elevated apoptosis before and during neural tube closure (Chi et al., 2005). On the other hand, some MAPKKK knockout mice, such as Ask1(-l-), Tp/2(-l-) and Map3k2 $(-/-)$, survive embryonic development and are born alive with no overt developmental defects. These mice, however, display different abnormalities when challenged by pathogen and stressors. The Ask1(-l-) embryonic fibroblasts are resistant to apoptosis induced by TNFa and $\mathrm{H}_{2} \mathrm{O}_{2}$ (Tobiume et al., 2001), the Tp/2(-/-) mice produce low levels of TNFa when treated by lipopolysaccharide (LPS) (Dumitru et al.,
2000), and the Map3k2(-/-) T cells show increased proliferation and susceptibility to apoptosis induced by T-cell receptor cross-linking (Guo et al., 2002). The gene knockout studies have therefore revealed unique functions of each MAPKKK in vivo, reinforcing the idea that MAPKKK provides specificity in signal transduction.

An additional piece of evidence supporting this view is from the MAP3K1 knockout mice. Genetic ablation of MAP3K1 results in a unique developmental defect of the ocular surface that has not been found in mice lacking any of the other MAPKKKs. Furthermore, detailed analyses of the MAP3K1 knockout mice lead to uncover an intertwining relationship between the MAPK cascades and c-Jun during development.

\section{MAP3K1 IN OCULAR SURFACE DEVELOPMENT}

MAP3K1 was originally identified as a mammalian homolog of the yeast MEK kinases, Byr2 and Ste11, involved in pheromone-induced mating (Lange-Carter et al., 1993). Although initially considered as an upstream activator of ERK, it soon became clear that MAP3K1 preferentially activates the JNK pathway, by interacting with and phosphorylating the JNK activator, MKK4 (Minden et al., 1994; Yan et al., 1994; Lin et al., 1995; Xia et al., 1998). MAP3K1 is in turn activated by various upstream signals, and intriguingly, each signal appears to be coupled with a distinct mechanism through the $\mathrm{N}$-terminal regulatory domain.

The N-terminal domain of MAP3K1 can bind to the SH3 domain of Grb2, resulting in the recruitment of the MAP3K1/ Grb2 complex by the Shc proteins to the activated EGF receptor and a transient plasma membrane localization of

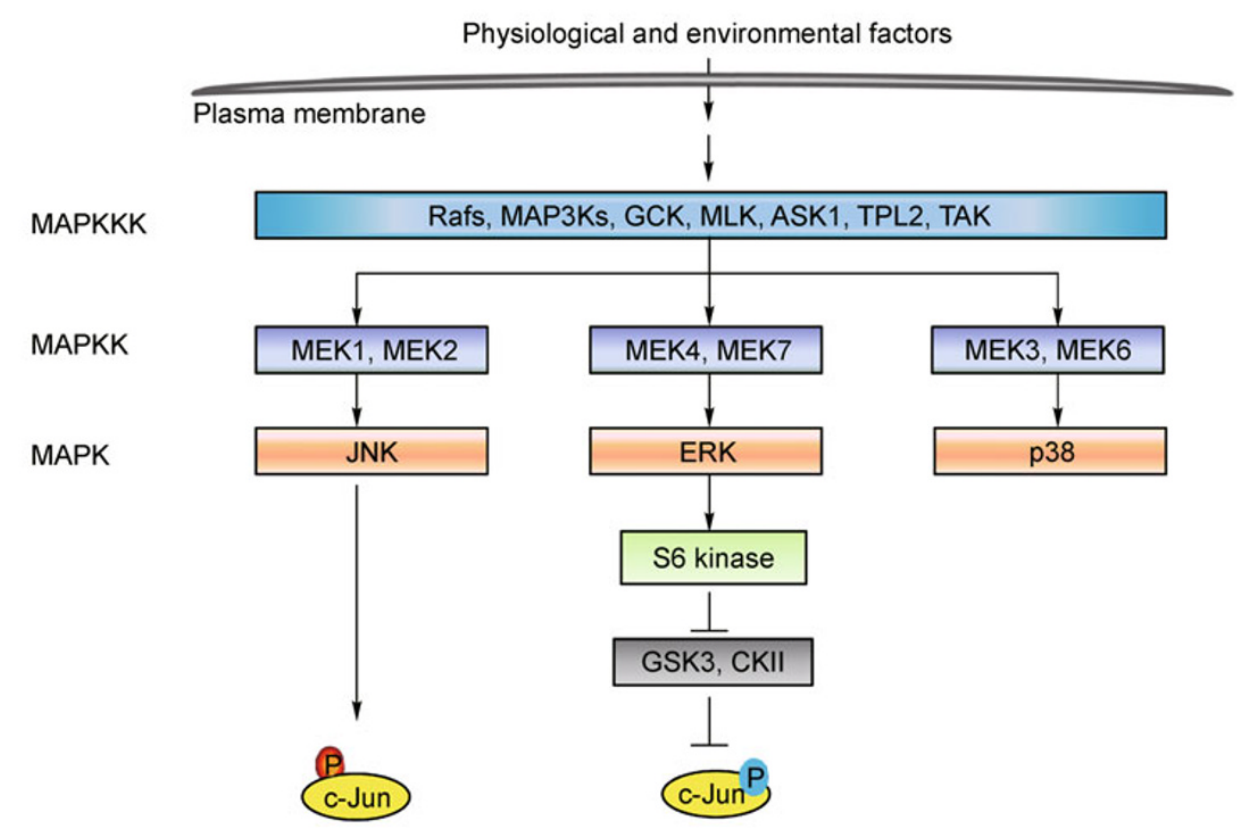

Figure 2. Signaling mechanisms of c-Jun phosphorylation. A diagram illustrating the MAP3K-MAP2K-MAPK signaling cascades, of which both the JNK and ERK cascades are involved in c-Jun phosphorylation. 
MAP3K1 (Pomérance et al., 1998). The membrane localized MAP3K1 undergoes conformational changes to initiate autoactivation, by which MAP3K1 phosphorylates its own threonine residues between the kinase subdomains VII and VIII (Deak and Templeton, 1997; Siow et al., 1997). The Nterminal domain of MAP3K1 also offers binding sites for RhoA, JNK and alpha-actinin, while its C-terminal kinase domain binds MKK4 (Xu et al., 1996; Xia et al., 1998; Christerson et al., 1999; Gallagher et al., 2004). Such scaffolding function allows MAP3K1 to organize a signaling complex to efficiently transmit the RhoA signal to the downstream MEK4-JNK pathway that is linked to the actin cytoskeleton. TGF $\beta$ may utilize such a mechanism to act through RhoA to control c-Jun phosphorylation and actin cytoskeleton (Atfi et al., 1997; Gallagher et al., 2004; Zhang et al., 2005). MAP3K1 can also interact with axin, mediating Wnt signal in JNK activation and planar polarity determination (Zhang et al., 1999). In addition, several protein kinases, including HPK, NIK, PKG and GLK, have been shown to directly associate with and phosphorylate MAP3K1 in vitro, acting as the possible upstream activators for MAP3K1 (Diener et al., 1997; Su et al., 1997; Soh et al., 2001).

Despite the fact that MAP3K1 has different regulatory mechanisms and numerous potential functions, its most striking in vivo role is the regulation of ocular surface morphogenesis. Mice lacking either the full-length MAP3K1 protein (MAP3K1-null or Map3k1(-l-)) or its kinase domain (Map3k1( $\triangle K D / \triangle K D)$ ) survive embryonic development and do not show overt developmental defects except for an "eyeopen at birth" (EOB) phenotype (Yujiri et al., 2000; Zhang et al., 2003).

Mammalian eye development involves a transient closure and re-opening of the eyelid (Findlater et al., 1993). In mice, eyelid development begins at embryonic day 13 (E13), when the surface ectoderm folds into the lid buds. The eyelid buds continue to grow towards the center of the ocular surface and by E15-E16.5, the epithelial cells at the tip of the eyelid start to elongate and migrate. Ultimately, the epithelium of upper and lower eyelid fuses to form a closed eyelid. Mouse eyelids remain closed between E16.5 and postnatal day 12, and thereafter the lid fusion breaks down as a consequence of epithelial cell apoptosis at the fusion junction, resulting in open eyelids. Mice are normally born with their eyelid closed, but those impaired in embryonic eyelid closure are born with EOB phenotype.

Genetic studies in mice have identified complex signal transduction processes involved in eyelid closure. Besides MAP3K1, embryonic eyelid closure depends on signals derived from WNT, Sonic hedgehog, BMP/Activin, FGF and EGF (Luetteke et al., 1993; Mine et al., 2005; Gage et al., 2008; Huang et al., 2009). In addition, eyelid closure requires the participation of a number of intracellular signaling kinases, such as $\mathrm{JNK}, \mathrm{ROCK}$ and $\mathrm{CDH} 1$, and nuclear transcription factors, such as c-Jun, Fra-2, FOXL2, SMAD and GRHL3
(McHenry et al., 1998; Li et al., 2003; Zenz et al., 2003; Zhang et al., 2003; Uda et al., 2004; Thumkeo et al., 2005; Takatori et al., 2008; Yu et al., 2008; Naoe et al., 2010). While how these factors are organized into a morphogenetic network for eyelid closure has not been well understood, molecular analyses of these mice have begun to unveil that some of the factors are organized into concerted signal transduction cascades.

\section{THE MAP3K1-C-JUN INTRACRINE REGULATORY LOOP FOR EYELID MORPHOGENESIS}

Studies centered on the Map3k1 knockout mice have led to the identification of a signaling pathway, assembled by several of the "eyelid closure" factors. This pathway is initiated by activin $B$, which binds to a specific cell surface receptor to activate MAP3K1. MAP3K1 in turn activates the MKK4-JNK cascade that further leads to c-Jun N-terminal phosphorylation (Takatori et al., 2008; Geh et al., 2011). Activation of this pathway is essential for embryonic eyelid closure, while its inadequate activation in mice lacking activin $B$ and MAP3K1 results in EOB. Furthermore, MAP3K1 is haploinsufficient for eyelid closure in Jnk1(-/-) and Jnk1(+/-) $J n k 2(+/-)$ mice, providing genetic evidence for the existence of a MAP3K1-JNK signaling axis in the regulation of eyelid development (Takatori et al., 2008).

Activation of the MAP3K1-JNK cascade promotes epithelial cell migration, which is required for the developing eyelid tip epithelial cells to move forward for eyelid closure (Yujiri et al., 2000; Zhang et al., 2003). One downstream event of the MAP3K1-JNK axis is the phosphorylation of c-Jun at its Nterminus, which leads to increased AP-1 activity and enhanced expression of AP-1 target genes, such as PAl-1 (Zhang et al., 2003; Takatori et al., 2008; Geh et al., 2011). Nonetheless, neither the transgenic mice expressing a phosphorylation site mutated c-Jun nor the Pai-1 knockout mice display EOB, raising the possibility that c-Jun $\mathrm{N}$-terminal phosphorylation and some of the AP-1 regulated genes are not involved in eyelid closure (Behrens et al., 1999; Providence and Higgins, 2004). The MAP3K1-JNK signaling cascades must have other downstream effectors to regulate developmental eyelid closure. For example, this pathway may be directly involved in the regulation of actin cytoskeleton reorganization, crucial for epithelial cell migration and eyelid closure (Zhang et al., 2003; Zhang et al., 2005).

On the other hand, ablation of c-Jun specifically in keratinocytes does not affect skin development, but it causes EOB (Li et al., 2003; Zenz et al., 2003). It has been proposed that C-Jun regulates the expression of EGFR or its ligand HBEGF, which are necessary for stimulating epithelial cell migration and embryonic eyelid closure (Grose, 2003). Correspondingly, genetic knockout of the EGFR itself or its ligands, TGFa and HB-EGF or of factors, such as FGF10 and GRHL3 that regulate ligand expression, all lead to impaired epithelial cell migration and the EOB phenotype (Fig. 3). 


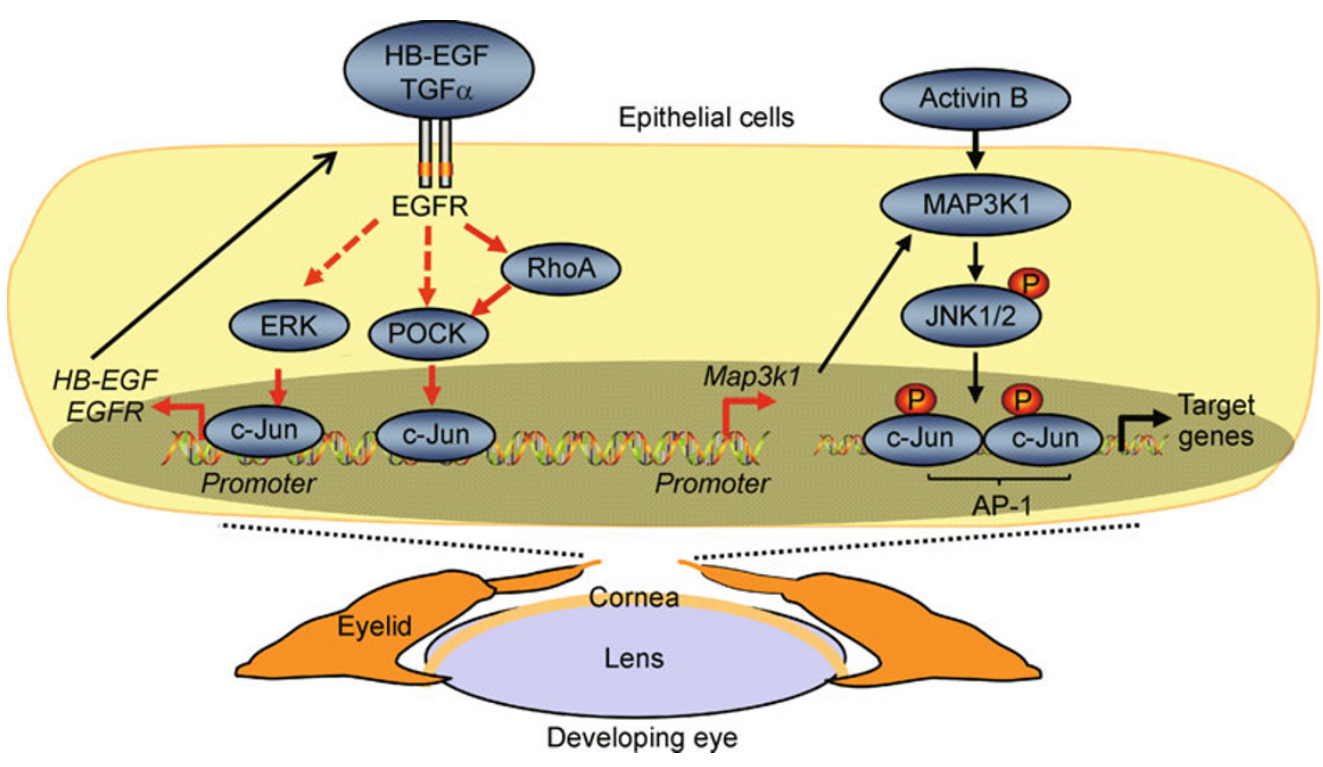

Figure 3. The EGFR, MAP3K1 and c-Jun intracrine regulatory loop for eyelid morphogenesis. A diagram illustrating crosstalks of C-Jun with EGFR and MAP3K1 signaling pathways in the leading edge epithelium of developing eyelids. In this scheme, C-Jun can act upstream of EGFR/HB-EGF to regulate their gene expression, while on the other hand, c-Jun acts downstream of EGFR to regulate MAP3K1 expression, whereas, MAP3K1 in turn is required for C-Jun phosphorylation and optimal AP-1 activity.

Recently, studies from our laboratory have identified yet another novel signaling scheme for eyelid development (Geh et al., 2011). We showed that activation of the EGFR by TGFa initiates signaling cascades involving ROCK/RhoA that lead to induction of c-Jun. c-Jun, acting in an N-terminal phosphorylation independent fashion, directly binds to the Map3k1 promoter. The TGFa/EGFR-RhoA/ROCK-c-Jun pathway converges on the Map3k1 promoter, resulting in elevated MAP3K1 expression. Once expressed, the enzyme activities of MAP3K1 are required to activate the JNK-C-Jun cascade. Thus, MAP3K1 and c-Jun form an intracrine regulatory loop, in which c-Jun controls MAP3K1 expression, while MAP3K1 in turn controls c-Jun N-terminal phosphorylation and AP-1 activity (Fig. 3).

Transient lid closure and reopening is a common morphogenetic event that also takes place in humans. Unlike in mice, however, eyelid closure and re-opening in humans is accomplished in utero, making its deficiency difficult to detect. Clinical diagnosis of eyelid closure defects and identification of possible associated developmental diseases present a challenge that may have to rely on a genetic approach for resolution. In this context, the genetic mouse models with an easily traceable phenotype have led to the identification of a great number of molecular players involved in eyelid development. This information may help to decipher the "genetic codes" underlying eyelid developmental defects in humans, leading to unravel the origins of congenital developmental disorders in children.

\section{CONCLUSION}

After two decades of extensive efforts, we have become fully aware of the complex nature of c-Jun regulation and function. Still, as originally believed, c-Jun is a transcription factor that binds to a relatively simple sequence in the regulatory domain of genes, but just its ability to bind DNA and activate AP-1driven promoters is insufficient to explain its role in diverse and sometimes opposing physiological processes, including proliferation, apoptosis, survival, tumorigenesis and tissue morphogenesis. C-Jun-mediated cell responses can be affected by many factors and are dependent on the abundance of c-Jun protein, its dimerization partners, and its interactions with other transcription factors, co-activators and co-repressors. It has become recognized that c-Jun interacts dynamically with signaling pathways and forms complex feedback and feed forward regulatory loops that are yet to be fully understood. Studies of eyelid development provide a peek view of such complexity, in which c-Jun acts as a molecular bridge connecting different signaling pathways.

Recently, global transcriptom profiling and complex molecular genetics have been used to provide additional understanding of C-Jun (Florin et al., 2004; Wolter et al., 2008; Biddie et al., 2011; Li et al., 2011). It is anticipated that future work, based on sophisticated genetic and molecular approaches, will be instrumental to delineate the multifaceted regulation and functions of c-Jun in vivo. 


\section{ACKNOWLEDGEMENTS}

We thank Dr. A. Puga, University of Cincinnati, for reading the manuscript. Work in our laboratory described in this paper is supported in part by a grant from the National Institutes of Health, NIH EY15227.

\section{ABBREVIATIONS}

AP-1, activator protein-1; ASK1, apoptosis-stimulated kinase 1; ASV 17, avian sarcoma virus 17; ATF, activating transcription factor; bZIP, basic leucine zipper; EOB, eye-open at birth; ERK, extracellular signal regulated kinase; GCK, germinal center kinase; GSK-3, glycogen synthase kinase 3; JNKs, c-Jun amino-terminal kinases; MAF, musculoaponeurotic fibrosarcoma; MAPKs, mitogen-activated protein kinases; MAP3K1, mitogen-activated protein kinase kinase kinase 1; MAPKKs, MAP kinase kinases; MAPKKKs, MAPKK kinases; MLK, mixed lineage kinases; TAK, TGF-beta-activated kinase; TPL2, tumor progression locus 2

\section{REFERENCES}

Adler, V., Polotskaya, A., Wagner, F., and Kraft, A.S. (1992). Affinitypurified c-Jun amino-terminal protein kinase requires serine/ threonine phosphorylation for activity. J Biol Chem 267, 17001-17005.

Angel, P., Allegretto, E.A., Okino, S.T., Hattori, K., Boyle, W.J., Hunter, T., and Karin, M. (1988a). Oncogene jun encodes a sequencespecific trans-activator similar to AP-1. Nature 332, 166-171.

Angel, P., Hattori, K., Smeal, T., and Karin, M. (1988b). The jun protooncogene is positively autoregulated by its product, Jun/AP-1. Cell 55, 875-885

Angel, P., and Karin, M. (1991). The role of Jun, Fos and the AP-1 complex in cell-proliferation and transformation. Biochim Biophys Acta 1072, 129-157.

Atfi, A., Djelloul, S., Chastre, E., Davis, R., and Gespach, C. (1997). Evidence for a role of Rho-like GTPases and stress-activated protein kinase/c-Jun N-terminal kinase (SAPK/JNK) in transforming growth factor beta-mediated signaling. J Biol Chem 272, 1429-1432.

Baker, S.J., Kerppola, T.K., Luk, D., Vandenberg, M.T., Marshak, D. R., Curran, T., and Abate, C. (1992). Jun is phosphorylated by several protein kinases at the same sites that are modified in serum-stimulated fibroblasts. Mol Cell Biol 12, 4694- 4705.

Behre, G., Whitmarsh, A.J., Coghlan, M.P., Hoang, T., Carpenter, C. L., Zhang, D.E., Davis, R.J., and Tenen, D.G. (1999). C-Jun is a JNK-independent coactivator of the PU.1 transcription factor. J Biol Chem 274, 4939-4946.

Behrens, A., Jochum, W., Sibilia, M., and Wagner, E.F. (2000). Oncogenic transformation by ras and fos is mediated by c-Jun Nterminal phosphorylation. Oncogene 19, 2657-2663.

Behrens, A., Sibilia, M., and Wagner, E.F. (1999). Amino-terminal phosphorylation of c-Jun regulates stress-induced apoptosis and cellular proliferation. Nat Genet 21, 326-329.

Bengal, E., Ransone, L., Scharfmann, R., Dwarki, V.J., Tapscott, S.J., Weintraub, H., and Verma, I.M. (1992). Functional antagonism between c-Jun and MyoD proteins: a direct physical association. Cell 68, 507-519.
Biddie, S.C., John, S., Sabo, P.J., Thurman, R.E., Johnson, T.A., Schiltz, R.L., Miranda, T.B., Sung, M.H., Trump, S., Lightman, S.L., et al. (2011). Transcription factor AP1 potentiates chromatin accessibility and glucocorticoid receptor binding. Mol Cell 43, 145-155.

Black, E.J., Street, A.J., and Gillespie, D.A. (1991). Protein phosphatase $2 \mathrm{~A}$ reverses phosphorylation of c-Jun specified by the delta domain in vitro: correlation with oncogenic activation and deregulated transactivation activity of $v$-Jun. Oncogene 6 , 1949-1958.

Bohmann, D. (1990). Transcription factor phosphorylation: a link between signal transduction and the regulation of gene expression. Cancer Cells 2, 337-344.

Bohmann, D., Bos, T.J., Admon, A., Nishimura, T., Vogt, P.K., and Tjian, R. (1987). Human proto-oncogene c-jun encodes a DNA binding protein with structural and functional properties of transcription factor AP-1. Science 238, 1386-1392.

Bos, T.J., Bohmann, D., Tsuchie, H., Tjian, R., and Vogt, P.K. (1988). V-jun encodes a nuclear protein with enhancer binding properties of AP-1. Cell 52, 705-712.

Boyle, W.J., Smeal, T., Defize, L.H., Angel, P., Woodgett, J.R., Karin, M., and Hunter, T. (1991). Activation of protein kinase $C$ decreases phosphorylation of c-Jun at sites that negatively regulate its DNAbinding activity. Cell 64, 573-584.

Chi, H., Sarkisian, M.R., Rakic, P., and Flavell, R.A. (2005). Loss of mitogen-activated protein kinase kinase kinase 4 (MEKK4) results in enhanced apoptosis and defective neural tube development. Proc Natl Acad Sci U S A 102, 3846-3851.

Christerson, L.B., Vanderbilt, C.A., and Cobb, M.H. (1999). MEKK1 interacts with alpha-actinin and localizes to stress fibers and focal adhesions. Cell Motil Cytoskeleton 43, 186-198.

Davis, R.J. (1994). MAPKs: new JNK expands the group. Trends Biochem Sci 19, 470-473.

Deak, J.C., and Templeton, D.J. (1997). Regulation of the activity of MEK kinase 1 (MEKK1) by autophosphorylation within the kinase activation domain. Biochem J 322, 185-192.

Dérijard, B., Hibi, M., Wu, I.H., Barrett, T., Su, B., Deng, T., Karin, M., and Davis, R.J. (1994). JNK1: a protein kinase stimulated by UV light and Ha-Ras that binds and phosphorylates the c-Jun activation domain. Cell 76, 1025-1037.

Devary, Y., Gottlieb, R.A., Lau, L.F., and Karin, M. (1991). Rapid and preferential activation of the c-jun gene during the mammalian UV response. Mol Cell Biol 11, 2804-2811.

Diener, K., Wang, X.S., Chen, C., Meyer, C.F., Keesler, G., Zukowski, M., Tan, T.H., and Yao, Z. (1997). Activation of the C-Jun N-terminal kinase pathway by a novel protein kinase related to human germinal center kinase. Proc Natl Acad Sci U S A 94, 9687-9692.

Dumitru, C.D., Ceci, J.D., Tsatsanis, C., Kontoyiannis, D., Stamatakis, K., Lin, J.H., Patriotis, C., Jenkins, N.A., Copeland, N.G., Kollias, G., et al. (2000). TNF-alpha induction by LPS is regulated posttranscriptionally via a Tpl2/ERK-dependent pathway. Cell 103, 1071-1083.

Ellenberger, T.E., Brandl, C.J., Struhl, K., and Harrison, S.C. (1992). The GCN4 basic region leucine zipper binds DNA as a dimer of uninterrupted alpha helices: crystal structure of the protein-DNA complex. Cell 71, 1223-1237.

Findlater, G.S., McDougall, R.D., and Kaufman, M.H. (1993). Eyelid development, fusion and subsequent reopening in the mouse. $J$ 
Anat 183, 121-129.

Florin, L., Hummerich, L., Dittrich, B.T., Kokocinski, F., Wrobel, G., Gack, S., Schorpp-Kistner, M., Werner, S., Hahn, M., Lichter, P., et al. (2004). Identification of novel AP-1 target genes in fibroblasts regulated during cutaneous wound healing. Oncogene 23, 7005-7017.

Franklin, C.C., McCulloch, A.V., and Kraft, A.S. (1995). In vitro association between the Jun protein family and the general transcription factors, TBP and TFIIB. Biochem J 305, 967-974.

Franklin, C.C., Sanchez, V., Wagner, F., Woodgett, J.R., and Kraft, A. S. (1992). Phorbol ester-induced amino-terminal phosphorylation of human JUN but not JUNB regulates transcriptional activation. Proc Natl Acad Sci U S A 89, 7247-7251.

Gage, P.J., Qian, M., Wu, D., and Rosenberg, K.I. (2008). The canonical Wnt signaling antagonist DKK2 is an essential effector of PITX2 function during normal eye development. Dev Biol 317, 310-324.

Gallagher, E.D., Gutowski, S., Sternweis, P.C., and Cobb, M.H. (2004). RhoA binds to the amino terminus of MEKK1 and regulates its kinase activity. J Biol Chem 279, 1872-1877.

Geh, E., Meng, Q., Mongan, M., Wang, J., Takatori, A., Zheng, Y., Puga, A., Lang, R.A., and Xia, Y. (2011). Mitogen-activated protein kinase kinase kinase 1 (MAP3K1) integrates developmental signals for eyelid closure. Proc Natl Acad Sci USA 108, 17349-17354.

Grose, R. (2003). Epithelial migration: open your eyes to c-Jun. Curr Biol 13, R678-R680.

Guo, Z., Clydesdale, G., Cheng, J., Kim, K., Gan, L., McConkey, D.J., Ullrich, S.E., Zhuang, Y., and Su, B. (2002). Disruption of Mekk2 in mice reveals an unexpected role for MEKK2 in modulating T-cell receptor signal transduction. Mol Cell Biol 22, 5761-5768.

Hai, T.W., Liu, F., Allegretto, E.A., Karin, M., and Green, M.R. (1988). A family of immunologically related transcription factors that includes multiple forms of ATF and AP-1. Genes Dev 2, 1216-1226.

Haluska, F.G., Huebner, K., Isobe, M., Nishimura, T., Croce, C.M., and Vogt, P.K. (1988). Localization of the human JUN protooncogene to chromosome region 1p31-32. Proc Natl Acad Sci U S A 85, 2215-2218.

Harshman, K.D., Moye-Rowley, W.S., and Parker, C.S. (1988). Transcriptional activation by the SV40 AP-1 recognition element in yeast is mediated by a factor similar to AP-1 that is distinct from GCN4. Cell 53, 321-330.

Hibi, M., Lin, A., Smeal, T., Minden, A., and Karin, M. (1993). Identification of an oncoprotein- and UV-responsive protein kinase that binds and potentiates the c-Jun activation domain. Genes Dev 7, 2135-2148.

Huang, J., Dattilo, L.K., Rajagopal, R., Liu, Y., Kaartinen, V., Mishina, Y., Deng, C.X., Umans, L., Zwijsen, A., Roberts, A.B., et al. (2009). FGF-regulated BMP signaling is required for eyelid closure and to specify conjunctival epithelial cell fate. Development 136, 1741-1750.

Hüser, M., Luckett, J., Chiloeches, A., Mercer, K., Iwobi, M., Giblett, S., Sun, X.M., Brown, J., Marais, R., and Pritchard, C. (2001). MEK kinase activity is not necessary for Raf-1 function. EMBO J 20, 1940-1951.

Johnson, G.L., and Lapadat, R. (2002). Mitogen-activated protein kinase pathways mediated by ERK, JNK, and p38 protein kinases.
Science 298, 1911-1912.

Johnson, R.S., van Lingen, B., Papaioannou, V.E., and Spiegelman, B.M. (1993). A null mutation at the c-jun locus causes embryonic lethality and retarded cell growth in culture. Genes Dev 7, 1309-1317.

Karin, M. (1991). Signal transduction and gene control. Curr Opin Cell Biol 3, 467-473.

Karin, M., Liu, Z., and Zandi, E. (1997). AP-1 function and regulation. Curr Opin Cell Biol 9, 240-246.

Kuan, C.Y., Yang, D.D., Samanta Roy, D.R., Davis, R.J., Rakic, P., and Flavell, R.A. (1999). The Jnk1 and Jnk2 protein kinases are required for regional specific apoptosis during early brain development. Neuron 22, 667-676.

Kyriakis, J.M., Banerjee, P., Nikolakaki, E., Dai, T., Rubie, E.A., Ahmad, M.F., Avruch, J., and Woodgett, J.R. (1994). The stressactivated protein kinase subfamily of c-Jun kinases. Nature 369 , 156-160.

Lamph, W.W., Wamsley, P., Sassone-Corsi, P., and Verma, I.M. (1988). Induction of proto-oncogene JUN/AP-1 by serum and TPA. Nature 334, 629-631.

Landschulz, W.H., Johnson, P.F., and McKnight, S.L. (1988). The leucine zipper: a hypothetical structure common to a new class of DNA binding proteins. Science 240, 1759-1764.

Lange-Carter, C.A., Pleiman, C.M., Gardner, A.M., Blumer, K.J., and Johnson, G.L. (1993). A divergence in the MAP kinase regulatory network defined by MEK kinase and Raf. Science 260, 315-319.

Lee, W., Mitchell, P., and Tjian, R. (1987). Purified transcription factor AP-1 interacts with TPA-inducible enhancer elements. Cell 49, 741-752.

Li, G., Gustafson-Brown, C., Hanks, S.K., Nason, K., Arbeit, J.M., Pogliano, K., Wisdom, R.M., and Johnson, R.S. (2003). c-Jun is essential for organization of the epidermal leading edge. Dev Cell 4, 865-877.

Li, M., Ge, Q., Wang, W., Wang, J., and Lu, Z. (2011). c-Jun binding site identification in K562 cells. J Genetics Genomics (Yi chuan xue bao) 38, 235-242.

Lin, A., Frost, J., Deng, T., Smeal, T., al-Alawi, N., Kikkawa, U., Hunter, T., Brenner, D., and Karin, M. (1992). Casein kinase II is a negative regulator of c-Jun DNA binding and AP-1 activity. Cell 70, 777-789.

Lin, A., Minden, A., Martinetto, H., Claret, F.X., Lange-Carter, C., Mercurio, F., Johnson, G.L., and Karin, M. (1995). Identification of a dual specificity kinase that activates the Jun kinases and p38Mpk2. Science 268, 286-290.

Luetteke, N.C., Qiu, T.H., Peiffer, R.L., Oliver, P., Smithies, O., and Lee, D.C. (1993). TGF alpha deficiency results in hair follicle and eye abnormalities in targeted and waved-1 mice. Cell 73, 263-278.

Maki, Y., Bos, T.J., Davis, C., Starbuck, M., and Vogt, P.K. (1987). Avian sarcoma virus 17 carries the jun oncogene. Proc Natl Acad Sci U S A 84, 2848-2852.

McHenry, J.Z., Leon, A., Matthaei, K.I., and Cohen, D.R. (1998). Overexpression of fra- 2 in transgenic mice perturbs normal eye development. Oncogene 17, 1131-1140.

Minden, A., Lin, A., McMahon, M., Lange-Carter, C., Derijard, B., Davis, R.J., Johnson, G.L., and Karin, M. (1994). Differential activation of ERK and JNK mitogen-activated protein kinases by Raf-1 and MEKK. Science 266, 1719-1723. 
Mine, N., Iwamoto, R., and Mekada, E. (2005). HB-EGF promotes epithelial cell migration in eyelid development. Development 132 , 4317-4326.

Mordret, G. (1993). MAP kinase kinase: a node connecting multiple pathways. Biology of the cell / under the auspices of the European Cell Biology Organization 79, 193-207.

Nakamura, T., Datta, R., Kharbanda, S., and Kufe, D. (1991). Regulation of jun and fos gene expression in human monocytes by the macrophage colony-stimulating factor. Cell Growth Differ 2, 267-272.

Naoe, H., Araki, K., Nagano, O., Kobayashi, Y., Ishizawa, J., Chiyoda, T., Shimizu, T., Yamamura, K., Sasaki, Y., Saya, H., et al. (2010). The anaphase-promoting complex/cyclosome activator Cdh1 modulates Rho GTPase by targeting p190 RhoGAP for degradation. Mol Cell Biol 30, 3994-4005.

Nikolakaki, E., Coffer, P.J., Hemelsoet, R., Woodgett, J.R., and Defize, L.H. (1993). Glycogen synthase kinase 3 phosphorylates Jun family members in vitro and negatively regulates their transactivating potential in intact cells. Oncogene 8, 833-840.

Papavassiliou, A.G., Chavrier, C., and Bohmann, D. (1992). Phosphorylation state and DNA-binding activity of c-Jun depend on the intracellular concentration of binding sites. Proc Natl Acad Sci U S A 89, 11562-11565.

Pertovaara, L., Sistonen, L., Bos, T.J., Vogt, P.K., Keski-Oja, J., and Alitalo, K. (1989). Enhanced jun gene expression is an early genomic response to transforming growth factor beta stimulation. Mol Cell Biol 9, 1255-1262.

Pomérance, M., Multon, M.C., Parker, F., Venot, C., Blondeau, J.P., Tocqué, B., and Schweighoffer, F. (1998). Grb2 interaction with MEK-kinase 1 is involved in regulation of Jun-kinase activities in response to epidermal growth factor. J Biol Chem 273, 24301-24304.

Providence, K.M., and Higgins, P.J. (2004). PAI-1 expression is required for epithelial cell migration in two distinct phases of in vitro wound repair. J Cell Physiol 200, 297-308.

Quantin, B., and Breathnach, R. (1988). Epidermal growth factor stimulates transcription of the c-jun proto-oncogene in rat fibroblasts. Nature 334, 538-539.

Rauscher, F.J. 3rd, Cohen, D.R., Curran, T., Bos, T.J., Vogt, P.K., Bohmann, D., Tjian, R., and Franza, B.R. Jr. (1988a). Fosassociated protein p39 is the product of the jun proto-oncogene. Science 240, 1010-1016.

Rauscher, F.J. 3rd, Voulalas, P.J., Franza, B.R. Jr, and Curran, T. (1988b). Fos and Jun bind cooperatively to the AP-1 site: reconstitution in vitro. Genes Dev 2, 1687-1699.

Ryder, K., Lau, L.F., and Nathans, D. (1988). A gene activated by growth factors is related to the oncogene v-jun. Proc Natl Acad Sci U S A 85, 1487-1491.

Ryder, K., and Nathans, D. (1988). Induction of protooncogene c-jun by serum growth factors. Proc Natl Acad Sci U S A 85, 8464-8467.

Schlesinger, T.K., Fanger, G.R., Yujiri, T., and Johnson, G.L. (1998). The TAO of MEKK. Front Biosci 3, D1181-D1186.

Schumacher, M.A., Goodman, R.H., and Brennan, R.G. (2000). The structure of a CREB bZIP.somatostatin CRE complex reveals the basis for selective dimerization and divalent cation-enhanced DNA binding. J Biol Chem 275, 35242-35247.

Shaulian, E., and Karin, M. (2002). AP-1 as a regulator of cell life and death. Nat Cell Biol 4, E131-E136.
Sherman, M.L., Stone, R.M., Datta, R., Bernstein, S.H., and Kufe, D. W. (1990). Transcriptional and post-transcriptional regulation of cjun expression during monocytic differentiation of human myeloid leukemic cells. J Biol Chem 265, 3320-3323.

Short, N.J. (1987). Regulation of transcription. Are some controlling factors more equal than others? Nature 326, 740-741.

Siow, Y.L., Kalmar, G.B., Sanghera, J.S., Tai, G., Oh, S.S., and Pelech, S.L. (1997). Identification of two essential phosphorylated threonine residues in the catalytic domain of Mekk1. Indirect activation by Pak3 and protein kinase C. J Biol Chem 272, 7586-7594.

Smeal, T., Binetruy, B., Mercola, D., Grover-Bardwick, A., Heidecker, G., Rapp, U.R., and Karin, M. (1992). Oncoprotein-mediated signalling cascade stimulates c-Jun activity by phosphorylation of serines 63 and 73. Mol Cell Biol 12, 3507-3513.

Smeal, T., Binetruy, B., Mercola, D.A., Birrer, M., and Karin, M. (1991). Oncogenic and transcriptional cooperation with $\mathrm{Ha}$-Ras requires phosphorylation of c-Jun on serines 63 and 73. Nature 354, 494-496.

Soh, J.W., Mao, Y., Liu, L., Thompson, W.J., Pamukcu, R., and Weinstein, I.B. (2001). Protein kinase $G$ activates the JNK1 pathway via phosphorylation of MEKK1. J Biol Chem 276, 16406-16410.

Su, Y.C., Han, J., Xu, S., Cobb, M., and Skolnik, E.Y. (1997). NIK is a new Ste20-related kinase that binds NCK and MEKK1 and activates the SAPK/JNK cascade via a conserved regulatory domain. EMBO J 16, 1279-1290.

Sutherland, C., Renaux, B.S., McKay, D.J., and Walsh, M.P. (1994). Phosphorylation of caldesmon by smooth-muscle casein kinase II. J Muscle Res Cell Motil 15, 440-456.

Takatori, A., Geh, E., Chen, L., Zhang, L., Meller, J., and Xia, Y. (2008). Differential transmission of MEKK1 morphogenetic signals by JNK1 and JNK2. Development 135, 23-32.

Thumkeo, D., Shimizu, Y., Sakamoto, S., Yamada, S., and Narumiya, S. (2005). ROCK-I and ROCK-II cooperatively regulate closure of eyelid and ventral body wall in mouse embryo. Genes Cells 10 , 825-834.

Tobiume, K., Matsuzawa, A., Takahashi, T., Nishitoh, H., Morita, K., Takeda, K., Minowa, O., Miyazono, K., Noda, T., and Ichijo, H. (2001). ASK1 is required for sustained activations of JNK/p38 MAP kinases and apoptosis. EMBO Rep 2, 222-228.

Toda, T., Shimanuki, M., and Yanagida, M. (1991). Fission yeast genes that confer resistance to staurosporine encode an AP-1-like transcription factor and a protein kinase related to the mammalian ERK1/MAP2 and budding yeast FUS3 and KSS1 kinases. Genes Dev 5, 60-73.

Uda, M., Ottolenghi, C., Crisponi, L., Garcia, J.E., Deiana, M., Kimber, W., Forabosco, A., Cao, A., Schlessinger, D., and Pilia, G. (2004). Foxl2 disruption causes mouse ovarian failure by pervasive blockage of follicle development. Hum Mol Genet 13, 1171-1181.

Wagner, E.F., Schonthaler, H.B., Guinea-Viniegra, J., and Tschachler, E. (2010). Psoriasis: what we have learned from mouse models. Nat Rev Rheumatol 6, 704-714.

Wolter, S., Doerrie, A., Weber, A., Schneider, H., Hoffmann, E., von der Ohe, J., Bakiri, L., Wagner, E.F., Resch, K., and Kracht, M. (2008). C-Jun controls histone modifications, NF-kappaB recruitment, and RNA polymerase II function to activate the ccl2 gene. Mol Cell Biol 28, 4407-4423. 
Xia, Y., Wu, Z., Su, B., Murray, B., and Karin, M. (1998). JNKK1 organizes a MAP kinase module through specific and sequential interactions with upstream and downstream components mediated by its amino-terminal extension. Genes Dev 12, 3369-3381.

Xu, S., Robbins, D.J., Christerson, L.B., English, J.M., Vanderbilt, C. A., and Cobb, M.H. (1996). Cloning of rat MEK kinase 1 cDNA reveals an endogenous membrane-associated 195-kDa protein with a large regulatory domain. Proc Natl Acad Sci U S A 93, 5291-5295.

Yan, M., Dai, T., Deak, J.C., Kyriakis, J.M., Zon, L.I., Woodgett, J.R., and Templeton, D.J. (1994). Activation of stress-activated protein kinase by MEKK1 phosphorylation of its activator SEK1. Nature 372, 798-800.

Yang, J., Boerm, M., McCarty, M., Bucana, C., Fidler, I.J., Zhuang, Y., and Su, B. (2000). Mekk3 is essential for early embryonic cardiovascular development. Nat Genet 24, 309-313.

Yu, Z., Bhandari, A., Mannik, J., Pham, T., Xu, X., and Andersen, B. (2008). Grainyhead-like factor Get1/Grhl3 regulates formation of the epidermal leading edge during eyelid closure. Dev Biol 319, $56-67$.

Yujiri, T., Ware, M., Widmann, C., Oyer, R., Russell, D., Chan, E.,
Zaitsu, Y., Clarke, P., Tyler, K., Oka, Y., et al.(2000). MEK kinase 1 gene disruption alters cell migration and c-Jun $\mathrm{NH}$-terminal kinase regulation but does not cause a measurable defect in NF-kappa B activation. Proc Natl Acad Sci U S A 97, 72727277.

Zenz, R., Scheuch, H., Martin, P., Frank, C., Eferl, R., Kenner, L., Sibilia, M., and Wagner, E.F. (2003). c-Jun regulates eyelid closure and skin tumor development through EGFR signaling. Dev Cell 4, 879-889.

Zhang, L., Deng, M., Parthasarathy, R., Wang, L., Mongan, M., Molkentin, J.D., Zheng, Y., and Xia, Y. (2005). MEKK1 transduces activin signals in keratinocytes to induce actin stress fiber formation and migration. Mol Cell Biol 25, 60-65.

Zhang, L., Wang, W., Hayashi, Y., Jester, J.V., Birk, D.E., Gao, M., Liu, C.Y., Kao, W.W., Karin, M., and Xia, Y. (2003). A role for MEK kinase 1 in TGF-beta/activin-induced epithelium movement and embryonic eyelid closure. EMBO J 22, 4443-4454.

Zhang, Y., Neo, S.Y., Wang, X., Han, J., and Lin, S.C. (1999). Axin forms a complex with MEKK1 and activates c-Jun $\mathrm{NH}(2)$-terminal kinase/stress-activated protein kinase through domains distinct from Wnt signaling. J Biol Chem 274, 35247-35254. 